\title{
Quasi-resonant cavity enhanced photodetector with a subwavelength grating
}

\author{
Gongqing Li (李宫清), Xiaofeng Duan (段晓峰)，Weifang Yuan (袁纬方), Yongqing Huang (黄永清), Kai Liu (刘 凯), and \\ Xiaomin Ren (任晓敏) \\ State Key Laboratory of Information Photonics and Optical Communications, Beijing University of Posts and Telecommunications, Beijing 100876, China
}

*Corresponding author: xfduan@bupt.edu.cn

Received November 10, 2021 | Accepted December 2, 2021 | Posted Online January 4, 2022

\begin{abstract}
We designed a tunable wavelength-selective quasi-resonant cavity enhanced photodetector (QRCE-PD) based on a highcontrast subwavelength grating [SWG]. According to simulation results, its peak quantum efficiency is $93.2 \%$, the $3 \mathrm{~dB}$ bandwidth is $33.5 \mathrm{GHz}$, the spectral linewidth is $0.12 \mathrm{~nm}$, and the wavelength-tuning range is $28 \mathrm{~nm}$ (1536-1564 nm). The QRCE-PD contains a tunable Fabry-Perot (F-P) filtering cavity (FPC), a symmetrical SWG deflection reflector (SSWG-DR), and a built-in p-i-n photodiode. The FPC and the SSWG-DR form an equivalent multi-region F-P cavity together by multiple mutual mirroring, which makes the QRCE-PD a multi-region resonant cavity enhanced photodetector. But, QRCE-PD relies on the multiple-pass absorption enhanced effect to achieve high quantum efficiency, rather than the resonant cavity enhanced effect. This new photodetector structure is significant for the application in the dense wavelength division multiplexing systems.
\end{abstract}

Keywords: photodetector; quasi-resonant cavity; subwavelength grating; ultra-narrow linewidth.

DOI: 10.3788/COL202220.031301

\section{Introduction}

High speed ${ }^{[1]}$, high quantum efficiency, narrow spectral linewidth $^{[2-6]}$, and broad wavelength-tuning range $e^{[4-7]}$ are the critical performances for a photodetector applied in dense wavelength division multiplexing (DWDM) systems ${ }^{[8]}$. Due to the limitation of inherent efficiency bandwidth product, traditional normal-incidence photodetectors cannot achieve high speed and high quantum efficiency simultaneously. The resonant cavity enhanced photodetector (RCE-PD) can break this restriction by inserting the absorption region into the FabryPerot (F-P) resonant cavity ${ }^{[9-11]}$. However, the absorption effect will reduce the quality factor of the resonator, so that the spectral linewidth of the RCE-PD will be broadened. For a conventional RCE-PD, the spectral linewidth is commonly wider than $20 \mathrm{~nm}$, which is inapplicable for DWDM systems.

In order to narrow the linewidth of the photodetector, we either filter the incident light by using an external filter or integrate the filtering cavity ${ }^{[12]}$ with a photodiode. The external filter will increase the complexity of the optical system, so that several integrated parallel-mirrors multi-cavities resonance enhanced device structures were proposed ${ }^{[13-15]}$. But, there are optical coupling problems among these cavities. The decoupling condition exists, but it is very harsh, which requires higher fabrication precision. The multi-cavities structure still behaves like an RCEPD with filtering effects, and its wavelength-tuning range is limited by the spectral response envelope of the RCE-PD.
With the rise of the planar dielectric materials subwavelength grating (SWG $)^{[16-27]}$, the integration, miniaturization, and advancement of optoelectronics is promoted by combining functional SWG optical elements with optoelectronic devices. People can design SWGs to form a specific phase distribution condition, to control the incident light, and thus to realize the transmission, reflection $^{[17-19]}$, resonance ${ }^{[20]}$, deflection ${ }^{[21,22]}$, focusing ${ }^{[23,24]}$, splitting ${ }^{[25,26]}$, polarization, etc. All of these extraordinary features are significant for integration and application with optoelectronic devices in the field of optoelectronics and optical communications ${ }^{[16-27]}$.

In this paper, we proposed a new decoupling method, a tunable wavelength-selective quasi-RCE-PD (QRCE-PD) based on highcontrast SWG, which is composed of InP/InGaAs materials and operates at a $1550 \mathrm{~nm}$ long wavelength, as shown in Fig. 1. This photodetector contains a tunable F-P filtering cavity (FPC $)^{[12]}$, a symmetrical SWG deflection reflector (SSWG-DR) ${ }^{[21,22,27]}$ and a built-in p-i-n photodiode. According to the simulation results, the QRCE-PD can obtain a peak quantum efficiency of $93.2 \%$, a $3 \mathrm{~dB}$ bandwidth of $33.5 \mathrm{GHz}$, a spectral linewidth of $0.12 \mathrm{~nm}$, and a tuning range of $28 \mathrm{~nm}$ simultaneously.

\section{Structure Design of QRCE-PD}

For the design of the FPC, it is necessary to achieve narrow linewidth and high reflectivity at oblique incidence. The FPCs we 

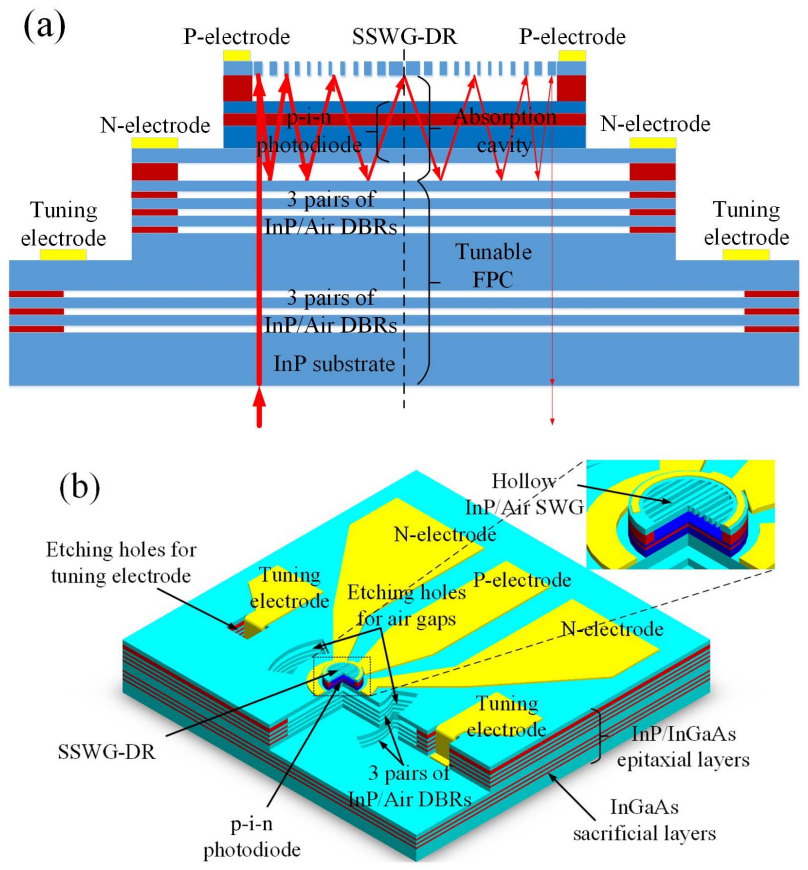

Fig. 1. (a) Schematic structure and (b) schematic layout of the QRCE-PD.

designed are made of two distributed Bragg reflector (DBR) mirrors. The DBR mirror contains three pairs of InP/air quarterwave stacks ${ }^{[11]}$. There is an InP cavity layer with a thickness of two effective wavelengths between the two DBRs. There are two tuning electrodes deposited on the cavity layer, which can be tuned by using carrier injection and thermal effects. The FPC is designed by using TFCalc software. As shown in Fig. 2, owing to the high-contrast refractive index of InP/air (3.167 and 1, separately), our designed FPC has an ultra-narrow linewidth of $0.12 \mathrm{~nm}$ and achieves $>97 \%$ reflectivity at $>3^{\circ}$ oblique incidence.

For the built-in photodiode, in order to pursue a higher frequency response speed, we temporarily ignore the quantum

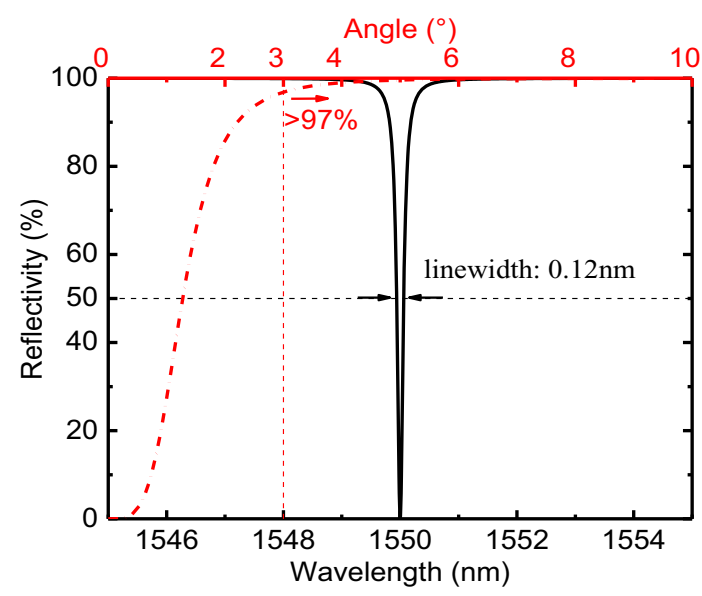

Fig. 2. Reflectivity of the FPC as a function of wavelength at normal incidence (black solid line) and as a function of incident angle at the $1550 \mathrm{~nm}$ wavelength (red dash dot line).
Table 1. Layer Structure of the Embedded p-i-n Photodiode.

\begin{tabular}{lccc}
\hline Name & Material & Doping $\left(\mathrm{cm}^{-3}\right)$ & Thickness $(\mathrm{nm})$ \\
\hline P layer & InGaAsP & $3 \times 10^{18}$ & 300 \\
Absorption layer & InGaAs & $1 \times 10^{15}$ & 400 \\
Spacing layer & InGaAsP & $2 \times 10^{15}$ & 1500 \\
N layer & InP & $3 \times 10^{18}$ & 300 \\
\hline
\end{tabular}

efficiency and adopt the absorption layer thickness of only $400 \mathrm{~nm}$. The layer structures used in this paper are given in Table 1 . At $-3 \mathrm{~V}$ bias, the $3 \mathrm{~dB}$ bandwidth of the device can reach $33.5 \mathrm{GHz}$.

With respect to designing a functional SWG optical element, it is a common way to arrange grating bars or blocks with appropriate phase and diffraction efficiency to form a specific phase profile. Herein, a grating reflector with $3^{\circ}$ deflection angle is designed and simulated by using COMSOL Multiphysics, whose reflectivity is $97 \%$, as shown in Fig. 3(a). The structure parameters of this grating are shown in Fig. 3(b), and its thickness is fixed at $0.5 \mu \mathrm{m}$. Then, we mirrored this grating horizontally to form the SSWG-DR. The SSWG-DR has an inclined-ridge reflection phase plane, which makes it equivalent to an inclined-ridge reflector.

The design of our QRCE-PD is based on InP/InGaAs materials for long wavelength devices and uses an InP/air gap structure and hollow InP/air grating structure. The main challenge in the fabrication of our device is the selective etching of the air gaps and the hollow InP/air SWG. The hollow grating structure was realized many times by the research team of ChangHasnain $^{[7,18,19]}$. The multi-gap DBR was realized by our lab in $2004^{[11]}$. So, we believe that our QRCE-PD can be realized. Referring to the schematic layout shown in Fig. 1(b), we will firstly fabricate the typical $\mathrm{p}-\mathrm{i}-\mathrm{n}$ photodetector mesa and cover it by photoresist. Then, two holes will be opened at both sides of the mesa and extended vertically down to the InP cavity layer of
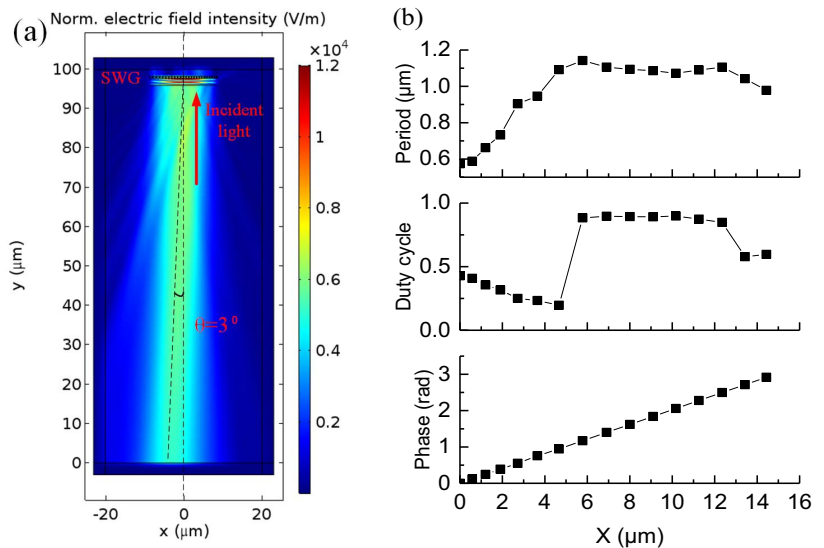

Fig. 3. (a) Electric field intensity distribution and (b) structure parameters of the SWG reflector. 
the FPC by standard photolithography and nonselective acid etching. Tuning electrodes will be deposited into the two holes by the electron beam vapor deposition method. Next, another two holes will be opened at both sides and extended down to the InP substrate by the same method. Selective lateral etching into the InGaAs sacrificial layers around these two holes will be carried out with selective etchant to form air gaps. Finally, we will define the grating by electron beam lithography (EBL) and form the hollow grating by the same selective etching.

\section{Theoretical Model of QRCE-PD}

The FPC, combined with the SSWG-DR, constitutes an inclined-ridge resonant cavity. Then, the $\mathrm{p}-\mathrm{i}-\mathrm{n}$ photodiode is embedded in it to form the QRCE-PD. In Ref. [25], the authors demonstrated that the inclined-ridge cavity is a multi-region F-P resonant cavity. QRCE-PD adopts this inclined-ridge resonant cavity structure but uses FPC instead of a bottom mirror. Figure 4(a) shows the equivalent inclined-ridge cavity structure of the QRCE-PD. By using the same method as in Ref. [25], QRCE-PD can be equivalent to a multi-region F-P cavity by multiple mutual mirroring. Figure 4 (b) depicts the mirroring schematic diagram of region 1 , in which the actual and equivalent optical paths are both shown, and an equivalent F-P cavity is clearly presented. The equivalent F-P cavities of other regions can also be presented in the same way.

Figure 5 shows the equivalent multi-region F-P cavity structure of the QRCE-PD. The conclusions from Ref. [25] indicate that the optical path and width of each region are determined by the cavity length $H$ and deflection angle $\theta$. Therefore, the number of resonant regions contained in a QRCE-PD depends on its

(a)

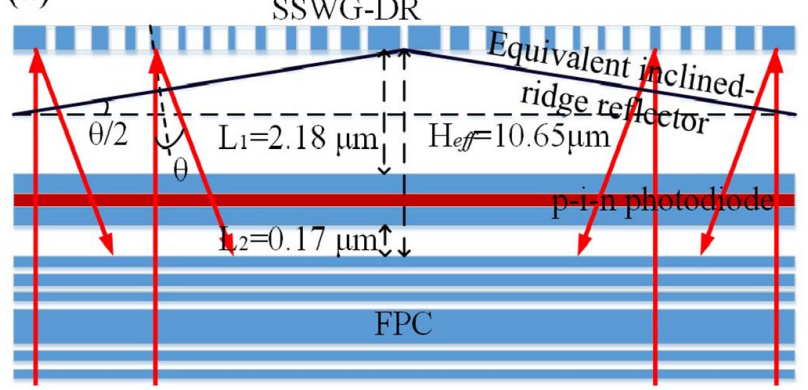

(b)

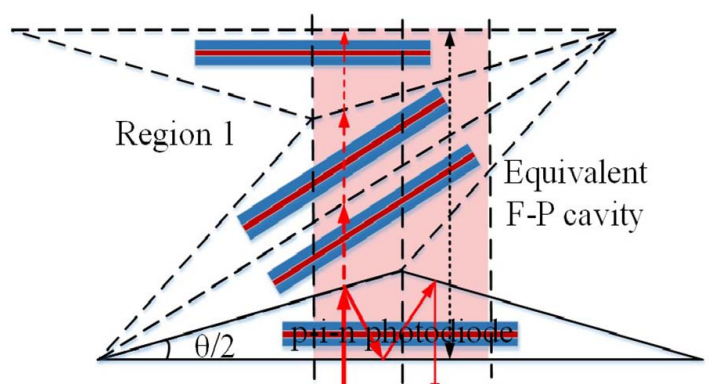

Fig. 4. (a) Equivalent inclined-ridge cavity structure of the QRCE-PD and (b) schematic diagram of forming region 1 by multiple mutual mirroring.

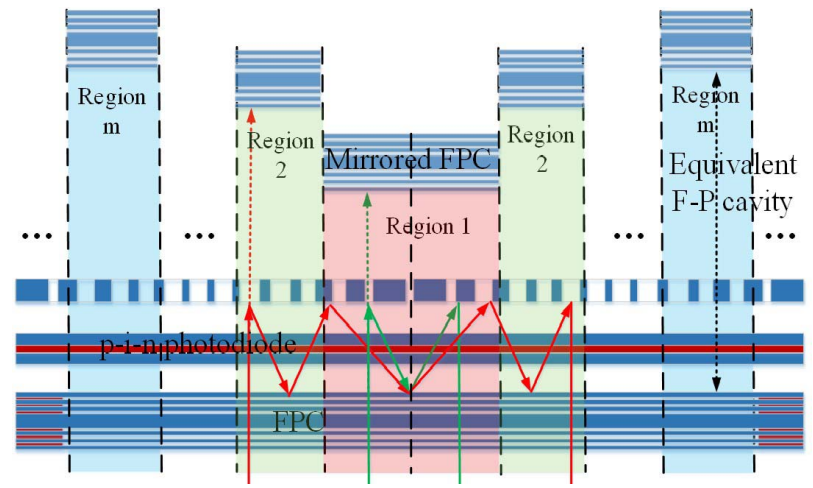

Fig. 5. Equivalent multi-region F-P cavity structure of the QRCE-PD, which forms a multi-region RCE-PDs structure together with a built-in photodetector.

$H$ and $\theta$. Of course, the size and material of the embedded p-i-n photodiode also affect the optical structure within the cavity, thus affecting the distribution of resonant regions. Here, the cavity parameters of our designed QRCE-PD are also shown in Fig. 4(a). Its effective cavity length $H_{\text {eff }}$ is $10.65 \mu \mathrm{m}$.

In any region of QRCE-PD, a photodetector, which is placed in an F-P cavity, is a typical RCE-PD. For a conventional RCE$\mathrm{PD}$, as employed from Ref. [10], under the assumption that all of the photo-generated carriers contribute to the detector current, the quantum efficiency $\eta$ could be expressed as

$$
\eta=\frac{\left(1+R_{2} e^{-\alpha d}\right)\left(1-R_{1}\right)\left(1-e^{\alpha d}\right)}{1-2 \sqrt{R_{1} R_{2}} e^{-\alpha d} \cos (\varphi)+R_{1} R_{2} e^{-2 \alpha d}},
$$

where $R_{1}, R_{2}$ are the reflectivity of the top mirror and end mirror, respectively, $\varphi$ is the total phase shift caused by the optical path of the resonant cavity, and $\alpha$ and $d$ are the absorption coefficient and thickness of the active layer. The $\eta$ is enhanced and maximized when $\varphi=2 n \pi(n=1,2,3, \cdots)$ and $R_{1}=R_{2} e^{-2 \alpha d}$. Similarly, in the resonant cavity enhanced (RCE) structure of the QRCE-PD, the top mirror is the FPC and the end mirror is the mirror image of the FPC, which means $R_{2}=R_{1}$. Thus, the only way to maximize $\eta$ is to make $R_{1}$ equal zero. In this case, the RCE structure of QRCE-PD degenerates into a conventional photodetector without interface reflection, and thus Eq. (1) can be simplified as

$$
\eta=1-e^{\alpha d}
$$

Fortunately and coincidentally, in the equivalent multi-region F-P cavity of the QRCE-PD, the ideal reflectivity of the FPC at the operating wavelength is zero, and the incident light will pass through the absorption layer several times when in any region. In region $m$, the incident light will propagate through the absorption layer $4 m$ times, which means that the $d$-thick absorber acts like a $4 m d$-thick absorber. So, the quantum efficiency in region $m$ is

$$
\eta_{m}=1-e^{4 m \alpha d}
$$

The total quantum efficiency of the QRCE-PD should be the weighted average of the quantum efficiency of each region, 


$$
\eta=k_{1} \eta_{1}+k_{2} \eta_{2}+\ldots+k_{m} \eta_{m}
$$

where $k_{m}$ is the ratio of the incident optical power in the region $m$ to the total incident optical power.

\section{Simulation Results and Discussions}

We simulated the spectral response of the QRCE-PD mentioned above by using the wave optics mode of COMSOL Multiphysics, which is based on the finite element method (FEM). In Fig. 6(a), the normalized electric field intensity distribution is shown, and the regions of our designed QRCE-PD are also identified. We know that the electric field intensity in the FPC is much stronger than that in the absorption cavity, which is consistent with the actual situation, because, in the absorption cavity, the incident light is absorbed in large quantities, while in the FPC the incident light is resonated and filtered.

Under the assumption that all of the photo-generated carriers contribute to the detector current, the spectral responses of a p-i-n photodetector, an RCE-PD, and our QRCE-PD are simulated and shown in Fig. 6(b). For comparison, all three devices use the same active photodiode, which is mentioned above. The quantum efficiency of the $\mathrm{p}-\mathrm{i}-\mathrm{n}$ photodetector is only $18.9 \%$. The peak value of the RCE-PD is near unity, but its spectral linewidth at full width at half-maximum (FWHM) is $15.9 \mathrm{~nm}$. As

(a)

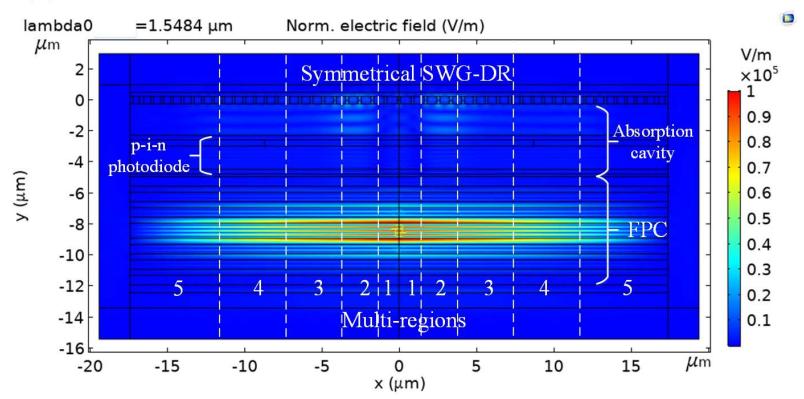

(b)

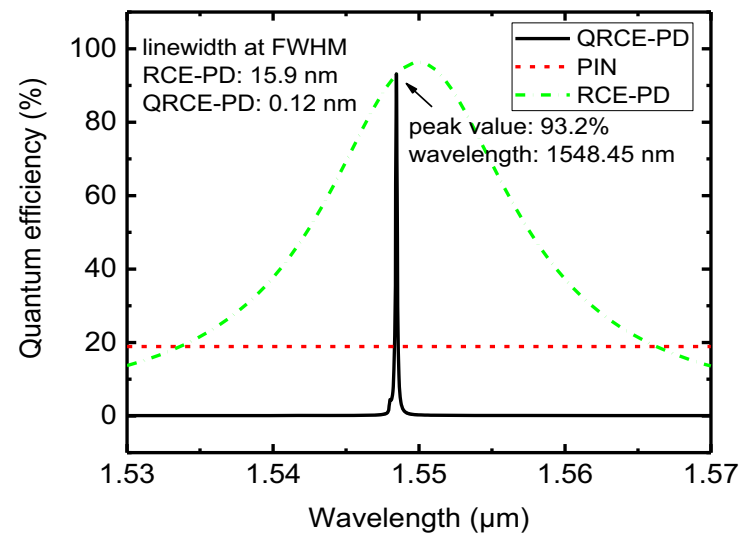

Fig. 6. (a) Normalized electric field intensity distribution and region distribution of the QRCE-PD. (b) Simulated spectral response of QRCE-PD, conventional RCE-PD, and $\mathrm{p}-\mathrm{i}-\mathrm{n}$ photodetector
Table 2. Theoretical Parameters of Each Absorption Region.

\begin{tabular}{lccc}
\hline Region $\boldsymbol{m}$ & Width $(\boldsymbol{\mu} \mathbf{m})$ & $\boldsymbol{k}_{\boldsymbol{m}}$ & $\boldsymbol{\eta}_{\boldsymbol{m}}$ \\
\hline 1 & 1.12 & 0.067 & 0.663 \\
2 & 2.23 & 0.134 & 0.887 \\
3 & 3.33 & 0.201 & 0.962 \\
4 & 4.43 & 0.267 & 0.987 \\
5 & 5.51 & 0.332 & 0.996 \\
\hline Total width & 16.62 & Total $\eta$ & 0.950 \\
\hline
\end{tabular}

expected, the QRCE-PD not only achieves a quantum efficiency of $93.2 \%$, but also has the same linewidth $(0.12 \mathrm{~nm})$ as the FPC. In Table 2, we list the theoretical parameters of each region derived from Ref. [27] and Eq. (3), such as width, $k_{m}$, and $\eta_{m}$. Then, we can obtain the theoretical total quantum efficiency according to Eq. (4). It is $95.0 \%$, which is higher than the actual total quantum efficiency. By comparing the simulation results to the theoretical values, we consider that the main factors that affect the quantum efficiency are the leakage of light incident from the SSWG-DR and the FPC.

However, the peak wavelength of QRCE-PD is not $1550 \mathrm{~nm}$, but shifts to $1548.45 \mathrm{~nm}$ unexpectedly. We consider that this offset is caused by the layer structure of the inserted photodiode, which changes the reflectivity of the top mirror of the FPC and leads to the offset of the resonant filtering center of the FPC. But, the offset can be eliminated by adjusting the reflectivity of the bottom mirror of FPC or by tuning the equivalent cavity length of the FPC. Changing the reflectivity of the bottom mirror needs to redesign the DBR; instead, adjusting the refractive index of the cavity layer of the FPC can change the equivalent cavity length to tune the peak wavelength. Under the assumption that the refractive index tuning is not limited, the QRCE-PD can obtain a wavelength-tuning range of

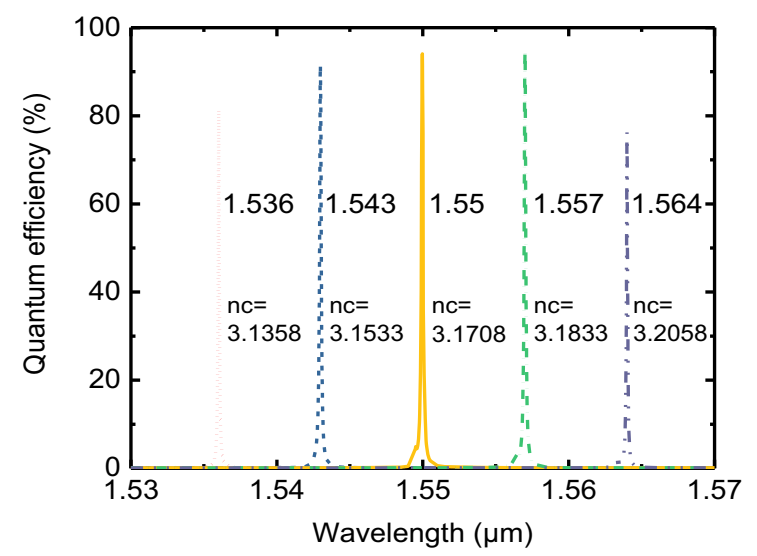

Fig. 7. Spectral response of QRCE-PD when tuning the refractive index of the cavity layer of the FPC. 


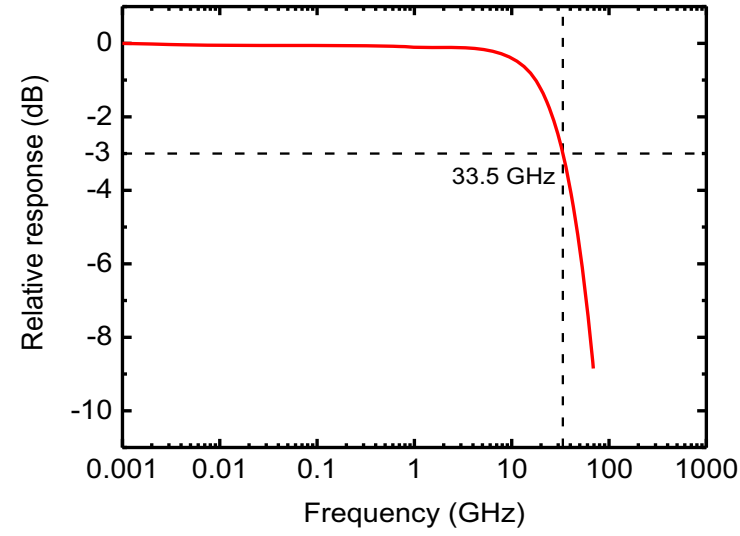

Fig. 8. Frequency response of the embedded $p$-i-n photodiode at $-3 \mathrm{~V}$ bias.

$28 \mathrm{~nm}(1536-1564 \mathrm{~nm})$ on the premise that the quantum efficiency is higher than $80 \%$, as shown in Fig. 7. The wide wavelength-tuning ability proves that the interpretation of the offset is reasonable. The distribution of the peak wavelengths does not conform to the spectral response envelope of the RCE-PD, which indicates that high quantum efficiency is not the result of the RCE effect, but relies on the multiple-pass absorption enhanced effect. That is why our device is named the QRCE-PD.

These results also verify that the FPC and the absorption cavity are decoupled and relatively independent. The FPC selects the wavelength, narrows the linewidth, and is tunable by carrier injection or thermal tuning. For the coupling of the incident light, the narrow linewidth also limits the incident angle variation. From Fig. 2, we know that the coupling efficiency will decrease dramatically when incident angle is larger than about $0.5^{\circ}$. Referring to previous experimental work on a tunable $\mathrm{FPC}^{[4-6]}$, the fabrication imperfection only shifts the central wavelength, and the effect on coupling efficiency and spectral linewidth is slight. Within the effective wavelength-tuning range, the spectral linewidth keeps narrow and stable, which is only slightly wider than the designed value. Furthermore, we can also design an additional circuit to feed the generated photocurrent back to the tuning circuit to stabilize the FPC. As for SSWG-DR, referring to Ref. [16], SWG has large fabrication imperfection tolerance. Besides, the wide wavelengthtuning range indicates that the SSWG-DR still performs well under the wavelength offset, because the wavelength-shift is also a kind of fabrication imperfection for an SWG. For the entire QRCE-PD, it is only needed to ensure that multiple mutual mirroring could be realized so that the QRCE-PD has good tolerance of fabrication imperfection.

The frequency response performance of the $\mathrm{p}-\mathrm{i}-\mathrm{n}$ photodetector used in this paper is simulated by Silvaco ATLAS software. The mesa diameter of this device is $32 \mu \mathrm{m}$. The result is shown in Fig. 8, where the $3 \mathrm{~dB}$ bandwidth is $33.5 \mathrm{GHz}$ under a reverse bias of $3 \mathrm{~V}$. The bandwidth is mainly limited by the resistancecapacity time constant. Since the spectral absorption and photoelectric conversion of semiconductor devices are two relatively independent physical mechanisms, and there is currently no
Table 3. Performance Parameters of Different Long Wavelength Devices.

\begin{tabular}{lccccc}
\hline \multicolumn{1}{c}{ Parameter } & RCE ${ }^{[1]]}$ & $\begin{array}{c}\text { Taper } \\
\text { cavity }{ }^{[5]}\end{array}$ & $\begin{array}{c}\text { Multi-cavities } \\
\text { RCE }^{[15]}\end{array}$ & QRCE \\
\hline Absorber thickness (nm) & 300 & 300 & 350 & 400 \\
Linewidth & Theoretical & - & - & 0.5 & 0.12 \\
$(\mathrm{~nm})$ & Experimental & $>60$ & 0.6 & 0.75 & - \\
Tuning range & Theoretical & - & - & - & 28 \\
$(\mathrm{~nm})$ & Experimental & - & 10.2 & - & - \\
Quantum & Theoretical & - & - & $80 \%$ & $93.2 \%$ \\
efficiency & Experimental & $59 \%$ & $70 \%$ & $70 \%$ & - \\
\hline
\end{tabular}

method to fully simulate the QRCE-PD device, we believe that it is reasonable to consider the optical response and frequency response of QRCE-PD separately. Therefore, in the future, the light detection part in QRCE-PD can be replaced by devices with higher response speed, such as the uni-traveling carrier photodetector.

In Table 3, for comparing the spectral response with our QRCE-PD, we list some performance parameters of several different long wavelength devices from references, such as quantum efficiency, spectral linewidth, and wavelength-tuning range. From this table, the device with the RCE structure ${ }^{[11]}$ can raise the quantum efficiency and narrow the linewidth slightly. But, for pursuing an ultra-narrow linewidth, an ultrathin absorber is needed in a high-quality resonant cavity, which is at the expense of the frequency response. As for the multicavities RCE-PD structure, there is a coupling problem between these cavities, and the decoupling condition is harsh, which is not conducive to wavelength tuning. The taper cavity structure is a way to decouple by setting a taper absorption cavity, but it suffers from the high light leakage and high fabrication difficulty. Our QRCE-PD structure is a new decoupling method that overcomes the disadvantages of the above structures we mentioned. For long wavelength devices, although the implementation scheme of QRCE-PD presented in this paper is challenging, its fabrication feasibility can be optimized.

\section{Conclusion}

In conclusion, we have proposed a tunable, high speed, high quantum efficiency, and ultra-narrow linewidth QRCE-PD with SWG, which can be equivalent to multi-region RCE-PDs by multiple mutual mirroring. The QRCE-PD structure is a new type of photodetector structure, whose most significant advantage is that it can greatly relieve the mutual restriction among quantum efficiency, response speed, and spectral linewidth. This structure can not only be applied to the DWDM system, but also provides a reference for the design of photodetectors with similar performance requirements in other optical fields. 


\section{Acknowledgement}

This work was supported in part by the National Key Research and Development Program of China (No. 2018YFB2200803).

\section{References}

1. A. Beling and J. C. Campbell, "InP-based high-speed photodetectors," J. Light. Technol. 27, 343 (2009).

2. J. Chen and Z. Zhou, "Ultranarrow band and high-quantum-efficiency photo response of Ge-on-Si photodetectors using cascaded-cavity structure," Appl. Phys. Lett. 89, 043126 (2006).

3. X. Duan, Y. Huang, H. Huang, X. Ren, Q. Wang, Y. Shang, X. Ye, and S. Cai, "Monolithically integrated photodetector array with a multistep cavity for multiwavelength receiving applications," J. Light. Technol. 27, 4697 (2009).

4. J. Lv, H. Huang, X. Ren, A. Miao, Y. Li, H. Song, Q. Wang, Y. Huang, and S. Cai, "Monolithically integrated long-wavelength tunable photodetector," J. Light. Technol. 26, 338 (2008).

5. H. Huang, X. Ren, X. Wang, H. Cui, W. Wang, A. Miao, Y. Li, Q. Wang, and Y. Huang, "Theory and experiments of a tunable wavelength-selective photodetector based on a taper cavity," Appl. Opt. 45, 8448 (2006).

6. X. Duan, Y. Huang, X. Ren, H. Huang, S. Xie, Q. Wang, and S. Cai, "Reconfigurable multi-channel WDM drop module using a tunable wavelength-selective photodetector array," Opt. Express 18, 5879 (2010).

7. M. C. Y. Huang, Y. Zhou, and C. J. Chang-Hasnain, "A nanoelectromechanical tunable laser," Nat. Photonics 2, 180 (2008).

8. G. Keiser, Fiber Optic Communications (Springer, 2021)

9. M. S. Ünlü and S. Strite, "Resonant cavity enhanced photonic devices," J. Appl. Phys. 78, 607 (1995).

10. H. S. Nalwa, Photodetectors and Fiber Optics (Elsevier, 2001).

11. H. Huang, Y. Huang, X. Wang, Q. Wang, and X. Ren, "Long wavelength resonant cavity photodetector based on InP/air-gap Bragg reflectors," IEEE Photon. Technol. Lett. 16, 245 (2004).

12. H. A. Macleod, Thin-Film Optical Filters, 4th ed. (CRC Press, 2010).

13. X. Ren and J. C. Campbell, "Theory and simulations of tunable two mirror and three-mirror resonant cavity photodetectors with a built in liquid-crystal layer," IEEE J. Quantum Electron. 32, 1903 (1996).
14. K. Liu, Y. Huang, and X. Ren, "Theory and experiments of a three cavity wavelength-selective photodetector," Appl. Opt. 39, 4263 (2000).

15. X. Duan, Y. Huang, X. Ren, W. Wang, H. Huang, Q. Wang, and S. Cai, "Long wavelength multiple resonant cavities RCE photodetectors on GaAs substrates," IEEE Trans. Electron Devices 58, 3948 (2011).

16. C. J. Chang-Hasnain and W. Yang, "High-contrast gratings for integrated optoelectronics," Adv. Opt. Photon. 4, 379 (2012).

17. M. C. Y. Huang, Y. Zhou, and C. J. Chang-Hasnain, "A surface-emitting laser incorporating a high-index-contrast subwavelength grating," Nat. Photonics 1, 119 (2007).

18. Y. Zhou, M. C. Y. Huang, and C. J. Chang-Hasnain, "Large fabrication tolerance for VCSELs using high contrast grating," IEEE Photon. Technol. Lett. 20, 434 (2008)

19. C. Chase, Y. Rao, W. Hofmann, and C. J. Chang-Hasnain, "1550 nm high contrast grating VCSEL," Opt. Express 18, 15461 (2010).

20. J. Hu, X. Liu, J. Zhao, and J. Zou, "Investigation of Fano resonance in compound resonant waveguide gratings for optical sensing," Chin. Opt. Lett. 15, 030502 (2017).

21. L. Carletti, R. Malureanu, J. Mørk, and I. S. Chung, "High-index-contrast grating reflector with beam steering ability for the transmitted beam," Opt. Express 19, 23567 (2011).

22. C. Ma, Y. Huang, X. Duan, and X. Ren, "High-transmittivity non-periodic sub-wavelength high-contrast grating with large-angle beam-steering ability," Chin. Opt. Lett. 12, 120501 (2014).

23. D. Fattal, J. Li, Z. Peng, M. Fiorentino, and R. G. Beausoleil, "Flat dielectric grating reflectors with focusing abilities," Nat. Photonics 4, 466 (2010).

24. T. Ma, X. Yuan, W. Ye, W. Xu, S. Qin, and Z. Zhu, "High focusing grating reflectors with TE-polarized normal incidence," Chin. Opt. Lett. 12, 020501 (2014).

25. G. Li, X. Duan, Y. Huang, K. Liu, and X. Ren, "Flat transmitted serratedphase high-contrast-index subwavelength grating beam splitter," Chin. Opt. Lett. 18, 110504 (2020).

26. C. Xiang, C. Zhou, W. Jia, and J. Wu, "Five-port beam splitter of a singlegroove grating," Chin. Opt. Lett. 16, 070501 (2018).

27. S. Zhang, X. Duan, G. Li, K. Liu, Y. Huang, and X. Ren, "High-reflectivity non-periodic sub-wavelength gratings with small-angle beam-steering ability and its application in Fabry-Perot cavity," Opt. Quantum Electron 50, 320 (2018). 